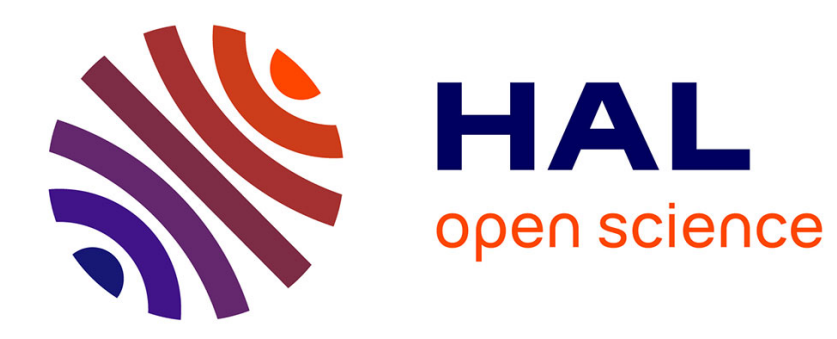

\title{
Temporalités sensorielles en quête de sens chez une adolescente
}

\author{
Anne Boisseuil
}

\section{To cite this version:}

Anne Boisseuil. Temporalités sensorielles en quête de sens chez une adolescente. Adolescence, 2014, 32 (4), pp.847-856. hal-01446953

\section{HAL Id: hal-01446953 \\ https://hal-amu.archives-ouvertes.fr/hal-01446953}

Submitted on 6 Feb 2017

HAL is a multi-disciplinary open access archive for the deposit and dissemination of scientific research documents, whether they are published or not. The documents may come from teaching and research institutions in France or abroad, or from public or private research centers.
L'archive ouverte pluridisciplinaire HAL, est destinée au dépôt et à la diffusion de documents scientifiques de niveau recherche, publiés ou non, émanant des établissements d'enseignement et de recherche français ou étrangers, des laboratoires publics ou privés. 


\section{Temporalités sensorielles en quête de sens chez une adolescente}

\section{Introduction}

Comment l'irruption de la génitalité au moment de l'adolescence, par le bouleversement des organisations psychiques infantiles, vient-elle mettre au présent des éléments sensoriels primaires ? Comment l'environnement contemporain de l'adolescent participe-t-il à cette dynamique ? Les liens précoces et les liens pubertaires s'appuient sur des rythmes primaires, socles de la construction de la continuité identitaire (Ph. Gutton, F. Marty) et la crise chez l'adolescent ouvre à des temporalités subjectives qui ne peuvent se comprendre sans tenir compte de l'effet de réponse chez les générations précédentes, dans un mouvement de l'un vers l'autre. L'organisation narcissique, infantile sont ainsi revisitées à l'aune d'une énigme pubertaire inquiétante, voire traumatique, éclatant la linéarité de la trame temporelle jusque là établie.

Grâce à la rencontre avec Nadia, une jeune fille de 15 ans venue consulter pour des troubles hallucinatoires, nous allons observer la manière dont elle utilise les voies de la sensorialité primaire pour élaborer sa sexualité génitale.

Nous examinerons la manière dont la symptomatologie de cette adolescente est une tentative de subjectivation de traumas précoces, d'éléments transgénérationnels et la mise en récit de la mère de cette jeune fille contribuera à mettre en sens. Ainsi, nous essayerons d'aborder cette situation clinique selon ses dynamiques temporelles où des modalités précoces, essentiellement sensori-rythmiques, sont traversées par la génitalité. Nous observerons comment Nadia les exprime par des formations psychiques originales dans un mouvement de conquête de son histoire subjective. 


\section{Présentation clinique}

Nadia a été suivie il y a quelques années pour dyspraxie dans le CMP où je travaille. Aujourd'hui adolescente, elle demande à parler à une femme de ses troubles hallucinatoires diagnostiqués comme des «Réminiscences visuelles et auditives au moment du coucher. » Cette dénomination est présentée comme une revendication consensuelle entre Nadia, sa mère ainsi que l'équipe médicale : le symptôme actuel est expliqué par des traumas précoces dont elle n'a pas souvenir. Ce mouvement semble tisser une «communauté de déni » (R. Kaës) qui a une fonction protectrice de cohésion familiale où le sexuel est nié : pas de différence des sexes, pas d'ambivalence.

Dans sa démarche de retour vers un lieu de soin de son enfance, Nadia effectue un travail de reprise psychique où la dysharmonie entre ses sensations et ses représentations s'exprime à nouveau mais transformée par le pubertaire et nous en soulignons deux aspects.

Le premier est la sexualisation. En effet, dans sa demande d'être entendue par une femme, elle signifie clairement que c'est de cette place sexuée qu'elle s'exprime, il n'y a pas de retour possible à un infantile non pubère.

Le deuxième est le vécu traumatique de l'énigme pulsionnelle. Son corps se révèle mystérieux, de nouvelles sensations émergent, jusque là voilées par le refoulement. Contrairement au symptôme infantile de dyspraxie, Nadia s'approprie la réponse événementielle de ses troubles car elle cherche à donner sens à ses éprouvés troublants. Ainsi, le passé s'agglutine sur le présent et la problématique narcissique prend le pas sur l’organisation œdipienne.

L'explication par le trauma détourne le regard de la sexualité génitale, elle procède d'une séduction qui masque celle du pulsionnel. Il fait l'objet d'un consensus familial, comme un leg traumatique qui la rattache à sa famille de naissance. Aujourd'hui, le consensus se 
retrouve dans le diagnostic de «réminiscence traumatique » où le trauma cristallise l'amalgame entre le sens subjectif des cauchemars de Nadia et l'explication désubjectivante imposée par l'extérieur.

«J'ai été témoin de tortures dont je n'ai pas le souvenir », me dira-t-elle dans les débuts de nos rencontres. Une mémoire rapportée dont elle a intériorisée des images, des fantasmes à partir de son organisation pulsionnelle, mais comment l'élaborer et pourquoi aujourd'hui ?

Dans le transfert, je participe pleinement à cette confusion en étant dépositaire d'un pan de son histoire dont elle ne m'aura pas parlé directement mais dont j'ai eu connaissance avant la première rencontre.

Elle m'a été présentée comme une jeune fille de 15 ans, d'origine slave, adoptée à deux ans par un couple français. Elle aurait assisté à des tortures sur des membres de sa famille et dans ce contexte traumatique, sa mère l'a alors confiée à un orphelinat à 18 mois, avec une lettre expliquant son geste.

Je retrouve alors l'écart entre les constructions imaginaires dans l'attente de l'objet et la rencontre avec celui-ci. Ainsi, j'imaginais une enfant traumatisée, une petite fille dyspraxique, une patiente qui demandait un thérapeute femme. Je portais en moi ces représentations imaginaires, plurielles qui seront déroutées par la rencontre avec la patiente «réelle »; une adolescente aux prises avec sa sexualité infantile, sa problématique narcissique mais aussi et surtout, sa sexualité génitale, aveuglante d'évidence et passée sous silence.

J'apprendrai que deux ans après l'adoption, le couple se séparera avec violence et que le père a rompu tout contact avec Nadia et son ex-femme. Cette dernière se consacrera à l'éducation de Nadia «sans homme » précisera-t-elle, jusqu'à récemment où elle a construit une relation stable avec un nouveau conjoint. Un nouvel environnement s'est désormais constitué puisqu'ils habitent ensemble. 
Nadia, à propos de son père, a des propos tranchés mais qui expriment une confusion dans ses identifications oedipiennes et narcissiques :

«S'il ne voulait pas de moi pourquoi il m'a adopté, si c'était pour nous quitter deux ans après? ». Nadia passe de «moi » à «nous », énonçant la différenciation fragile entre l'abandon du père et celui de l'homme.

Les résonances transgénérationnelles infiltrent les liens de filiations et les traumas semblent se répercuter d'une histoire à l'autre, rendant l'appropriation subjective de son histoire plus confuse. C'est ce qu'A. Konicheckis (2008) nomme comme « restes quantitatifs » pour désigner des objets non élaborés qui, parce qu'ils appartiennent à des générations précédentes provoquent des confusions générationnelles et nécessitent dans l'actuel un «étayage groupal » afin d'établir un travail de qualification et de symbolisation.

\section{Des liaisons sensorielles, temporalités en construction}

Nous prenons le parti de présenter les troubles hallucinatoires comme participant d'un processus de subjectivation et ce de fait nous les présentons en même temps que d'autres formations subjectives marquées par la mise au présent de la sensorialité.

\section{Délicatesse et violence}

Lorsque Nadia parle de ce qu'elle vit presque chaque nuit, elle dit avec précaution que «C'est une question délicate ». Quelques séances plus tard, elle reprendra cette formule qui, d'une question, deviendra un «sujet» délicat; ce que nous entendons comme la transformation de la sensorialité en sensualité....

«En fait, quand je vais me coucher, parfois avant de m'endormir...Je vois, je sais pas, c'est bizarre, des gens que je... qui se font torturer... je sais pas, si c'est moi qui le fait ou... mais pourtant j'aime pas faire du mal, je comprends pas, c'est pas moi...Je comprends pas, je veux pas mais c'est comme si je les déclenchais... mais c'est 
horrible... Je peux pas m'empêcher... C'est là devant mes yeux... je me vois les torturer, ce sang...c'est pas moi ça, moi je suis contre la violence... je sais pas...je préfère pas en parler...c'est moi et c'est pas moi...en plus c'est moi qui frappe et je me vois le faire et c'est horrible...en même temps, parfois, c'est moi qui suis la victime et parfois c'est moi le bourreau...je comprends pas....je... non je sais pas pourquoi je vois ça...non...je sais pas...c'est ...horrible... » dit-elle.

Sa voix est sourde, lente, éprouvante, l'atmosphère de la séance s'alourdit, se glace d'une lourde pesanteur. Le corps de Nadia s'affaisse, son regard se baisse et ses mots se tarissent. Le temps semble ralentir, devenir épais, il prend corps à mesure que celui de Nadia semble se désincarner, projetant sur le cadre de la séance ses perceptions non élaborées. La force de déliaison pulsionnelle opère ici et maintenant, la pensée se délite et le lien sensoriel devient la modalité transférentielle principale. L'atmosphère ainsi créée aplanie la distance entre l'objet et le sujet.

La sensorialité de ce récit est massive et me fait vivre avec Nadia une sidération de la pensée, un saisissement corporel que je partage dans l'instant et dont l'élaboration ne se fera que dans un second temps.

Son allure androgyne trouble car il est difficile de déterminer son sexe. Elle allie des vêtements sportifs masculins, une coupe très courte avec des signes féminins encore peu harmonieux comme un maquillage des yeux violent ainsi que de discrets bracelets...

Sa voix, par son rythme et son timbre, varie de manière radicale ; chaleureuse, enjouée lorsqu'elle parle de ses lectures, artificielle et monocorde, lorsqu'elle parle de son enfance.

«J'ai eu des troubles du sommeil et j'ai souffert de dyspraxie (...) Je sais que j'ai eu des expériences de sévices précoces mais dont je n'ai aucun souvenir. » Son ton détaché colore d'étrangeté l'échange. 
Son regard est lui aussi discordant, ce qui se traduit par un strabisme particulièrement marqué lorsqu'elle est prise par une tension émotionnelle, un conflit psychique. J'observe qu'il s'accentue tout particulièrement lorsque son discours verbal est en contradiction avec celui de son corps. Ses mots peuvent parler de joie alors que son visage, sa posture exprimeront de la douleur. Dans ces moments, je choisirais d'intervenir par des commentaires assez simples, essayant de rester au plus près de ce qu'elle dit. Le regard de Nadia se focalise alors sur moi, elle touche ses bracelets et prend le temps de réfléchir à ses réponses. Une mesure a été posée, modifiant la musicalité de l'échange.

L'accordage rythmique où le discours est d'abord reçu dans sa forme correspond à un besoin primaire de Nadia qui lui permet d'effectuer un travail de comodalité sensorielle oeil-voix et, dans le même mouvement, une mise en récit. Les aspects précoces de ce mode de fonctionnement chez une adolescente soulignent la complexité des niveaux subjectifs en cours. Cependant, la fragilité narcissique menace la continuité des liens intersubjectaux ce qui évoque un fonctionnement d'un temps d'avant la parole, un lien sensori-rythmique.

\section{De soi à l'autre, (des)accordages rythmiques}

En effet, dans la rythmicité de nos rencontres, sa présence aux séances est un enjeu : Nadia m'appelle si elle a quelques minutes de retard (que souvent elle n'a pas), en revanche, lorsqu'elle est absente aux séances, c'est sa mère qui me prévient. La continuité de son suivi sera entrecoupée par de nombreuses absences, toujours justifiées. Leur répétition résonne avec les ruptures et les écueils de la rencontre entre elle et ses objets primaires. Je suis alors celle qui l'attend, qu'elle met à distance puis retrouve et en éprouvant la permanence de l'objet dans son absence même, elle le transforme en objet interne.

De même, les séances répondent à un rythme fait de silences d'une qualité sensorielle forte, souvent denses, chargés d'éléments que je n'identifie pas mais qui m'imposent le silence. 
La continuité est ténue et je suis sensible à cette fragilité du lien où l'accueil de sa présence est aussi important que celui de son absence. Le mouvement rythmique dedans/dehors montre la quête de Nadia dans l'élaboration d'une délimitation subjective entre ces espaces.

La lecture est une autre formation subjective où Nadia découple ses sensations, elle cherche à maitriser ses mouvements pulsionnels et peut-être en élaborer de nouvelles liaisons.

«En ce moment je suis dans la poésie, je trouve fascinant le rythme des vers. En ce moment c'est Iphigénie! »

Je l'interroge alors sur ce qu'elle entend par « rythme » et elle me répond en insistant sur la sensorialité de ses lectures.

«Le livre me prend et je décroche plus...je sais que c'est pas bien (petit sourire gêné)...ça m'isole...mais j'y peux rien, quand un livre me prend...» dit-elle avec emphase. Elle me regarde avec intensité, contrôlant mon regard et s'assurant d'une limite entre elle et moi.

Lorsque je l'interroge sur la manière dont elle lit, je suis surprise : elle lit à voix haute dès qu'elle le peut, sans quoi les mots ne prennent pas sens.

«J'entends ma voix mais je l'entends pas, ça me berce. Si je lis pas comme ça je comprends rien, les mots n'ont pas de sens. »

C'est donc sur un arrière-plan rythmique auto-construit qu'elle accède au contenu symbolique du livre. Elle ne lit pas avec ses yeux mais avec ses oreilles afin de «retrouver la musicalité du poète » ainsi qu'elle le dit. Re-trouver l'intention désirante de cet objet externe garant de sa propre cohésion identitaire, tel pourrait être l'enjeu de cette re-trouvaille.

Ce mode de lecture la conduit à s'isoler du reste du monde, ses amis notamment, ce qu'elle dit regretter en partie. Ainsi, le plaisir tiré de ce type de lecture est multiple : il l'autorise à un certain retrait relationnel, lui permet de jouer avec la pensée d'un plaisir sensuel. Elle érotise la lecture en un jeu de dissimulation, transformation de ce que R. Roussillon appelle les jeux 
du cadre, le cacher-coucou. De plus, le livre a une forte valeur symbolique dans sa famille et dans son histoire personnelle. L'écrit devient ainsi un recours matériel et psychique à sa continuité identitaire.

Nadia questionne l'ambivalence pulsionnelle par la maitrise de l'objet et du «pouvoir de l'objet » sur elle. Cette position subjective passive, ainsi que le propose C. Chabert (2010), est fondamentale dans la constitution d'une continuité d'être. Par la manière de se construire un environnement sensori-rythmique, Nadia ne se contente pas de recréer des modalités d'appréhension du monde primaire, elle les crée ici et maintenant dans un mouvement de reprise originale. La transformation des éléments perceptifs en éléments symboliques nécessite donc la reprise et l'intériorisation d'un objet interne suffisamment stable. Or, les expériences primaires de Nadia ne semblent pas avoir permis son établissement pour que la résurgence du pubertaire ne les fragilise pas.

\section{Conquête d'une continuité}

Le conflit psychique cherche une résolution par la perception quand le refoulement fait défaut et le recours au percept devient la modalité subjective principale. Or de nouvelles sensations font jour pour cette adolescente qui cherche à les intégrer.

Dans ces diverses manifestations sensorielles, Nadia provoque son paysage sensoriel immédiat qui a pour centre et vecteur son corps ; pour exister elle a besoin de créer une sensation chez l'autre. Elle accroche l'autre dans ce qui s'apparente à des modalités intersubjectales primaires, basée sur l'immédiateté perceptive, sur la communication émotionnelle. Ainsi, même ses troubles hallucinatoires pourraient s'entendre comme une tentative de résolution de son paradoxe subjectif. Elle met en échec l'attente, refuse de se laisser traverser par la rêverie autrement qu'en maintenant un découplage sensoriel douloureux. Ce faisant, elle scande malgré tout une différence nécessaire entre jour et nuit, 
elle délimite le dedans du dehors, soi et l'autre et Nadia construit une temporalité historisante. Ces éléments sensoriels semblent déposés et Nadia pourrait se les être appropriés sans qu'ils lui appartiennent comme des restes de «traumatismes précoces » ainsi que le propose A. Konicheckis, (2008). Reprenant l'idée d'affiliation développée par ce dernier, nous suggérons que ces éléments sensoriels forment les fils épars d'une continuité identitaire en devenir, tissée aussi par les effets de rencontre avec ses objets contemporains. En effet, la réponse de ces objets présents participera à la reprise de rencontres passées, non élaborées par Nadia qui n'a pu les constituer que comme des objets sensoriels en négatifs.

Ainsi que Winnicott le présente dans son travail sur la quête de soi (1971), la créativité primaire peut devenir contrainte si l'objet de satisfaction, ici l'environnement maternel (qui comprend un père), ne peut être détruit dans le fantasme car trop absent dans la réalité. Ce manque crée une frustration prématurée qui génère du négatif hallucinatoire qui installe des zones de confusion primaire. Le partage en réflexivité de celle-ci est nécessaire pour en sortir ainsi que le propose R. Roussillon (2011) et consolider des aires de partage intersubjectales. C'est pourquoi nous avons souligné les aspects sensoriels du transfert mais aussi l'étayage sur la présence physique de la mère et la mise en récit qu'elle fera dans cet espace transférentiel.

Retracer une histoire pour Nadia aura été en effet nécessaire comme une dimension de son travail de subjectivation entre une mémoire rapportée, incorporée et une reprise plus différenciée de ses éprouvés actuels.

Dans l'histoire rapportée par la mère nous retiendrons les moments où l'émotion la submergeait, c'est à dire qui renvoie aussi à une forme de communication précoce où l'angoisse est le signal de l'entrée en communication. Si la scène du départ du père mobilise de la rage, celle où Nadia pouvait «sentir » la main de sa mère biologique provoque de 
l'effroi chez sa mère adoptive. Toutefois, la scène la plus intense reste celle de la découverte de Nadia à l'orphelinat.

La mère décrit Nadia comme une enfant au corps abîmé dont elle a ressenti l'appel. Le pathétique qu'elle décrit apaise la culpabilité inconsciente de la mère qui idéalise cette rencontre pour se laisser séduire par celle qu'elle reconnaît comme son enfant. Le peu de place à l'ambivalence, la mise en avant d'une sensorialité perceptive comme modalité de rencontre avec l'autre, sont autant de modalités subjectives que nous retrouvons chez Nadia.

D. Meltzer (2003) parle du « choc esthétique » entre la mère et le bébé comme une rencontre organisatrice de la pensée. De la qualification esthétique découle une expérience émotionnelle qui peut être entravée et alors des forces « anti-esthétiques » entravent la croissance psychique du sujet. Ainsi, la scène de la rencontre avec sa mère adoptive résonne comme un choc esthétique inversé où ce n'est pas la beauté de la mère qui est un choc pour l'enfant mais la douleur, la fragilité et les blessures de l'enfant qui séduisent la mère, ou plutôt les objets internes infantiles de celle-ci.

Nadia a intériorisé quelque chose de cet environnement fragile où ce qui est manifesté n'est pas toujours ajusté avec ce qui est éprouvé, où les mouvements affectifs ambivalents ne semblent que difficilement tolérés et conduisent à des organisations défensives primaires, comme le clivage.

A l'adolescence, les expériences précoces sont reprises sous différents organisateurs. C'est ainsi que la proposition plurifocale de l'intégration des liens sensoriels précoces d'A. Konicheckis nous aide à penser cette situation. La mise en récit des éléments historiques n’a pas valeur cathartique de manière magique mais s'inscrit dans une dynamique qui implique le lien transférentiel immédiat ainsi que l'implication de l'environnement affectif du sujet. Nadia s'accroche au percept et c'est une de ses façons d'attirer l'objet vers elle, ce qui rappelle la scène de son adoption. 
P. Gutton, lorsqu'il parle du processus pubertaire, amène l'idée d'une resexualisation du fonctionnement psychique qui peut faire rupture dans la continuité identitaire du sujet. En effet, c'est dans les temps d'avant que plongent les racines de ce qui se déploie aujourd'hui.

Ce temps d'avant la parole est ce avec quoi Nadia a élaboré ses premières formes de lien, sa première rencontre avec sa mère biologique, son environnement primaire, ses premiers mots dans une langue aux tonalités autres, à la musicalité qui la berçait. Or, cette période est aussi celle de la perte catastrophique de sa mère biologique, de la rencontre avec la mère française, celle de la parole et de l'écrit. En effet, la dissonance sensorielle de Nadia, portée par son corps, que ce soit dans son allure mais aussi dans l'intensité de son symptôme hallucinatoire peut s'entendre comme un message adressé à ses mères, celle de la petite enfance mais aussi bien la mère pubère.

Ainsi, Nadia envoie un signal de détresse, (au sens freudien «d'angoisse-signal »), sa mère peut recevoir et transformer. Elle ouvre pour elle-même mais aussi pour sa mère des espaces où les figures précoces et actuelles peuvent s'incarner chez un même objet. La mère pubère devient en même temps celle précoce, par « réflexivité » avec la simultanéité de l'intégration des éléments primaires et pubertaires chez Nadia.

\section{Conclusion}

La symptomatologie de Nadia nous a permis de réfléchir à la remobilisation d'éléments précoces au regard du pubertaire. Ainsi, nous avons observé que coexistaient des temporalités primaires, marquées par le rythme présent/absent, soi/non-soi avec d'autres temporalités où la perception du temps est tangible parce qu'un autre soutient l'inscription du sujet dans une histoire qu'il fonde et qui le fonde. C'est cette oscillation entre des dynamiques de mise en récit subjectif et d'autres plus isolés, dominés par le sensoriel, que nous retrouvons chez 
Nadia : un mélange hétérogène, par moment inquiétant mais qui renferme également toute une potentialité de réorganisation créative.

Cauchemars, manière de lire, déplacement sur le corps, ces formations subjectives sont comme de purs présents sensoriels. Eprouvées comme des éléments hors de soi, hors du temps, elles se manifestent sur le corps ou dans le lien à l'autre, en quête d'ancrages vers un récit historisable.

La ruse subjective de Nadia est d'aborder ce processus sous le couvert d'une explication liée à son passé traumatique. Par ce masque, elle aborde les irrésolus de ses énigmes subjectives, historiques mais aussi pulsionnelles. La mise en avant du sensoriel ouvre à une élaboration de sa sexualité génitale ainsi que des éléments transgénérationnels non élaborés, des restes traumatiques. L'environnement actuel est interpellé pour l'aider à qualifier ses éprouvés sensoriels, l'inédit du sensuel génital. Nous comprenons la quête de soi de Nadia comme la mise en œuvre des mouvements psychiques où les scènes passées, actuelles, internes et externes se regroupent et se réorganisent de façon originale pour chacun. Par la rupture avec la linéarité historique organisée par l'infantile, les éléments sensoriels de l'irruption pubertaire permettent l'intégration d'éléments énigmatiques inélaborés chez le sujet et potentiellement chez son environnement présent. Ainsi, la tolérance pour ces temporalités paradoxales conduit à des mouvements en double de subjectivation intégrative : du sujet vers l'objet et de l'objet vers le sujet.

Anne Boisseuil aboisseuil@gmail.com 24, Bd Gassendi 13012 Marseille CMP Hugues, $\mathrm{CH}$ Valvert 
35, bd Hugues

13012 Marseille 


\section{Bibliographie :}

BERTOLINI M. (2004). Le transfert de base en psychanalyse d'enfant et d'adolescent, Revue française de psychanalyse, 5, 68, pp. 1807-1822.

BOLOGNINI S. (2007). Le bar dans le désert, symétrie et asymétrie dans le traitement d'adolescents difficiles, Adolescence, 25, 1, pp. 133-144.

BOUBLI M., ELBEZ J.-C. (2010). Agirs et sensations à l'adolescence : appel à une mémoire implicite dans des modalités de lien à soi et à un objet externe en mal d'internalisation. Neuropsychiatrie de l'enfance et de l'adolescence, 58, pp. 234-240.

CHABERT C. (2010). L'essence du transfert. In : La psychanalyse des adolescents existe-telle ? Paris : PUF, pp. 83-103.

CICCONE A., FERRANT A. (2010). Indices de honte et dispositif psychothérapeutique, Dialogue, 4, 190, pp. 41 à 53.

CICCONE A. (2006). Partage d'expériences et rythmicité dans le travail de subjectivation, Carnet Psy, 5, 109, pp. 29 à 34.

FREUD S. (1919). L'inquiétante étrangeté. In : L'inquiétante étrangeté et autres essais, 1985, Paris : Gallimard, pp. 211-263.

FREUD S. (1930). Le malaise dans la culture, $3^{\text {ème }}$ éd. 1998, Paris : PUF.

GIMENEZ G. (2010). Halluciner, percevoir l'impensé. Paris : De Boeck.

GIVRE P. (2010). Préoccupation féminine adolescente. In : La psychanalyse des adolescents existe-t-elle?, Paris : PUF, pp. 121-145.

GUIGNARD F. (1996). Au vif de l'infantile. Lausanne : Delachaux et Niestlé.

GUTTON PH., BOURCET S. (2004). La cure sous traumatisme à l'adolescence, Cliniques méditerranéennes, 1, 69, pp. 187-207.

GUTTON PH. (1991). Le pubertaire. 2003. Paris : PUF.

HIRSCH D. (2009). Construction et interprétations à l'adolescence : du futur antérieur au passé recomposé, Adolescence, 27, 4, pp. 1027-1037.

KONICHECKIS A. (2009). Filiations sensorielles et processus de subjectivation, Le Divan familial, 1, 22, pp. 33 à 45.

KONICHECKIS A. (2008). De génération en génération : la subjectivation et les liens précoces. Paris : PUF.

KONICHECKIS A. (2003). Troubles psychiques liés aux interactions précoces, Spirale, 1, 25, pp. 149 à 160.

LACAN J. (1945). Le temps logique et l'assertion de logique anticipée. In : Ecrits 1, Paris : Seuil, 1999, pp. 195-211.

LEVINAS E. (1979). Le temps et l'autre. $9^{\text {ème }}$ éd. 2003, Paris : PUF.

MARTY F. (2001). La psychose pubertaire, une impasse du processus d'adolescence », Revue de psychothérapie psychanalytique de groupe, 36, 1, pp. 153-166. MELO I. (2009). Notes de lecture sur «Construction et interprétations à l'adolescence : du futur antérieur au passé recomposé » de Denis Hirsch, Adolescence, 27, 4, pp. 1039-1049.

MIJOLLA-MELLOR S. (2001). Le temps zizague et se chevauche, Topique, 1, nº74, pp. 716.

MELTZER D. (2003). Le transfert esthétique dans le processus psychanalytique. In: Psychothérapies de l'enfant et de l'adolescent. Paris : Bayard, pp. 345-355.

MOYANO O. (2010). Un cas de dépersonnalisation-déréalisation à l'adolescence : étude clinique des troubles dissociatifs. Neuropsychiatrie de l'enfance et de l'adolescence, 58 : 126-131.

RICHARD F. (2001). Le processus de subjectivation à l'adolescence. Paris : Dunod. 
ROUSSILLON R. (2011) Le transitionnel, le sexuel et la réflexivité. Paris : Dunod. ROUSSILLON R. (2004). La pulsion et l'intersubjectivité, Adolescence, 4, nº 50, p. 735 à 753.

ROUSSILLON R. (2010). Précarité et vulnérabilité identitaires à l'adolescence, Adolescence, 2, 72, pp. 241 à 252.

WINNICOTT D. W. (1971). La créativité et ses origines. In : Jeu et Réalité. Paris : PUF, pp. 91-119.

WINNICOTT D. W. (1962). L'adolescence. In : De la pédiatrie à la psychanalyse. Paris :

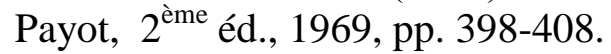

\section{Résumé :}

A partir de la clinique d'une adolescente reçue en consultation pour symptômes hallucinatoires, nous proposons une lecture de ses expressions subjectives selon une perspective temporelle. Nous axerons notre réflexion sur l'actualisation de liens primaires où la sensorialité peut devenir une voie de conquête vers la subjectivation. De ce fait, nous interrogerons la place de l'environnement actuel de l'adolescente comme étayage dans ce travail.

\section{Mots clés :}

Sensorialité, pubertaire, rythmes, temporalité

\section{Sensory temporalities in search of meaning with a teenager :}

Based on the clinical observation of a teenager who consulted for hallucinatory symptoms, I suggest an analysis of her subjective expressions depending on a temporal perspective. I will base my analysis on the recurrence of primary links in which the sensoriality might become a way toward subjectivism. Thus, I will interrogate the place of the current environment of the teenager as a basis for this study.

Key words :

Sensoriality, Pubertal, Rythm, Temporality

Temporalidades sensoriales en la búsqueda de significado en un adolescente :

A partir de la clínica de una adolescente recibida en consulta para síntomas alucinadores, proponemos una lectura de sus expresiones subjetivas según una perspectiva temporal. Orientaremos nuestra reflexión sobre la actualización de lazos primarios donde el sensorial puede hacerse una vía de conquista hacia el subjectivo. De hecho, interrogaremos el sitio del medio ambiente de la adolescente tomando como base de este trabajo.

Palabras claves :

Sensorialidad, pubertal, ritmos, temporalidad 